\title{
Monitoring of the Femtosecond Laser Micromachining Process of Materials Immersed in Water by Use of Laser-Induced Breakdown Spectroscopy
}

\author{
Aurimas Baskevicius*1, Ona Balachninaite*1, Mykolas Karpavicius*1, Simas Butkus*1, Domas Paipulas*1 and \\ Valdas Sirutkaitis*1 \\ ${ }^{* 1}$ Vilnius University, Faculty of Physics, Department of Quantum Electronics, Laser Research Center, \\ Sauletekio Ave. 10, Vilnius 10223, Lithuania \\ E-mail: aurimas.baskevicius@stud.ff.vu.lt
}

\begin{abstract}
Laser micromachining related with ablation can benefit from laser-induced breakdown spectroscopy (LIBS), as this not only allows for determining the chemical composition, but can also lead to an insight into the instant characteristics or quality of the micromachining. In this report, we present results on the application of LIBS in monitoring the micromachining of soda-lime glass immersed in water with femtosecond high repetition rate pulses.
\end{abstract}

DOI: $10.2961 /$ jlmn.2016.03.0018

Keywords: femtosecond pulse micromachining, laser-induced breakdown spectroscopy (LIBS), femtosecond ablation in water, glass cutting

\section{Introduction}

Ultrafast laser material processing is a quick, highly accurate, precise and flexible procedure that provides many advantages and benefits over other conventional processes [1]. Compared with long-pulse lasers, femtosecond laser pulses allow for extremely high peak irradiance intensities to be achieved (more than $10^{15} \mathrm{~W} / \mathrm{cm}^{2}$ ) with low pulse energies. At high intensities, nonlinear absorption takes place in transparent materials, allowing microprocessing to be conducted on or below the surface of these materials [2-5]. Moreover, for femtosecond lasers, due to the fact that the pulse duration is shorter than the time scales for electronphonon interaction, the laser affected zone is vaporized faster than the energy can be transferred to the surrounding areas, making extremely fine-feature pattering possible. All these features lead to better quality, increased machining efficiency and more reproducible and predictable machining results.

One of the important tasks during laser micromachining is the on-line monitoring of the process. Through monitoring, possible problems occurring during the micromachining process can be identified and corrected. Process monitoring and its consequent control are essential in laser micromachining and enhance the performance of the process by achieving the desired technical requirements for industrial and research purposes [6]. A wide range of monitoring methods is used for laser micromachining: acoustical [7], electrical [8], thermal [9], optical [10-12] and hybrid methods [13]. One more promising technique for monitoring laser processing is laser-induced breakdown spectroscopy (LIBS), which not only allows for identifying the chemical composition, but can also lead to an insight into the quality of the laser processing [14-21].

During laser-induced material breakdown, the intense laser pulses initiate an expanding plasma plume, which emits light from the atomic, ionized and molecular constituents of the sample. Hence, LIBS can provide analytical in- formation about the elemental composition of the material being ablated [22-24].

LIBS has several attractive features for sample analysis: real-time and in-situ measurement due to minimal sample preparation, multiple element detection capability regardless of the physical form and aggregation state of the material, local microanalysis capability and operational simplicity. LIBS has been used in a variety of applications such as depth profiling, soil characterization, surgical selective tissue removal, authentication of artworks and monitoring and control of laser material processing [14-21, 25-27].

Compared to LIBS based on conventional nanosecond laser systems, ultrafast LIBS is distinguished by increased stability and accuracy due to the distinctive features of ultrafast laser pulse generation and material interaction. This causes reduced plasma signal variation. The continuum emission of the ultrafast LIBS is lower and decays much faster than in the case of nanosecond LIBS. Moreover, in contrast to nanosecond pulses, during femtosecond laser ablation a lower plasma shielding effect occurs [28]. Taking all of the above into account, LIBS can be used as a reliable method for the monitoring and control of ultrafast laser micromachining processes.

In this paper, we present LIBS-based monitoring of glass cutting by employing a novel micromachining approach [29]. By applying a thin water layer on top of the glass sample and using a low NA objective, filaments can be created within the water layer and can be used for micromachining purposes. This results in high micromachining quality and throughput due to the spatial shaping of ultrashort pulses, and the cooling and cleaning features of the covering water. By using this micromachining method, axial translation is not required, while several-millimeter deep structures can be fabricated, and additional cooling is provided due to the added water layer, which prevents temperature and tensile stress gradient formation [30]. This work is focused on investigating the characteristics of LIBS spectra obtained during the above-mentioned femtosecond laser 
micromachining with different processing parameters (scanning speeds, pulse repetition rates, scanning algorithms etc.) in order to evaluate the suitability of this method for monitoring purposes.

\section{Experimental setup}

A schematic representation of the experimental setup is shown in Fig. 1. The experiments were performed using the Pharos ${ }^{\circledR}$ Yb:KGW femtosecond laser system (Light Conversion Ltd). A laser beam was positioned using a dual-axis galvanometric scanner (ScanLab Inc.), which was controlled by SCA fabrication software (Altechna Ltd). After the laser beam passed through the scanner, it was focused using an F- Theta lens ( $\mathrm{f}=100 \mathrm{~mm}$ ) on the sample, which was mounted on a three-dimensional motorized positioning stage (Standa Inc.) for precise positioning of the sample at the start.

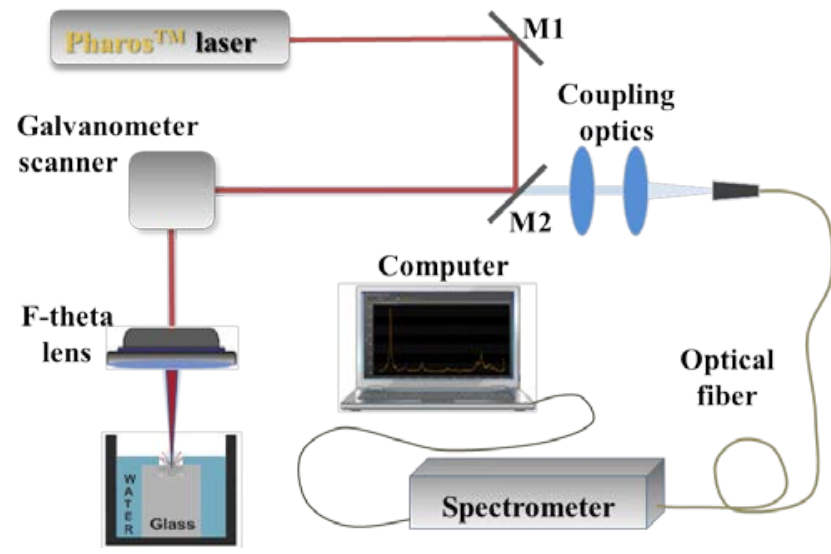

Fig. 1 LIBS-monitored femtosecond laser processing system schematics.

Micromachining was carried out on soda-lime glass of 1 millimeter thickness. The sample was covered with a thin (0.5 - $1.5 \mathrm{~mm}$ thick) water layer. The particular water layer thickness was chosen based on our previous experiments on glass cutting with high repetition rate femtosecond laser pulses using the same experimental setup. [29, 31]. The shortest cutting time for cutting $1 \mathrm{~mm}$ thick glass was obtained with a water layer thickness between $0.5 \mathrm{~mm}$ and 0.8 $\mathrm{mm}$. In contrast to the longer nanosecond or even picosecond laser pulses used for laser micromachining in the presence of water, and where a liquid layer thickness of at least several or even tens of millimeters could be used, in the case of femtosecond pulses, due to pulse filamentation and huge intensities, multiphoton absorption is an important loss mechanism, which drastically decreases the pulse energy reaching the sample for a larger thickness of water [32]. Our optimization experiments for high repetition rate pulses with large pulse energy [31] showed that the water thickness must be small, and in reality it becomes vapor at the fastest cutting conditions. On the other hand, water thickness must be at least $0.3 \mathrm{~mm}$ for cooling the sample and cleaning grooves and holes with cavitation bubbles. The observed plasmas or excited species in reality exceeded the $\sim 0.8 \mathrm{~mm}$ thickness of water layer and were not totally immersed in the water, but for optimization of the processing the important factors are processing quality and processing speed. So we worked on these relations using LIBS signal monitoring. Tap water was used for the experiments and the desired thickness of the layer was formed by submerging the glass sample in a reservoir. The thickness of this layer was measured by a CCD camera mounted on the side of the reservoir and if necessary refilled manually.

Typical micromachining parameters were chosen as follows: average pulse power - 3-5 W, pulse duration - $280 \mathrm{fs,}$ repetition rate $-60 \mathrm{kHz}-100 \mathrm{kHz}$, wavelength $-1030 \mathrm{~nm}$, focal spot diameter- $20 \mu \mathrm{m}$.

The plasma emission during laser ablation, which occurs above the sample surface at ambient temperature and pressure, is initially collected by the F-Theta lens, then passes through the galvanometer scanner and goes to the mirror M2, which is used for laser radiation direction, as it has high reflection coefficient for $1030 \mathrm{~nm}$ but is transparent for LIBS radiation in some spectral ranges. In our case, we mainly used a 400- $700 \mathrm{~nm}$ spectral range for LIBS investigations. After mirror M2, the transmitted LIBS radiation was collected by a pair of lenses and directed to the optical fiber that was connected to the multichannel spectrometer (AvaSpec USB2-DT, Avantes Inc.). The spectrometer was synchronized with the laser system and integration time was $100 \mathrm{~ms}$. In all cases, mainly one channel of the spectrometer in the spectral range $550-660 \mathrm{~nm}$ was used for our experiments, since one of the strongest soda-lime glass spectral lines $\mathrm{Na} I$ is located at a wavelength of $589 \mathrm{~nm}$. This channel has a 2048-element CCD array and provides an optical resolution $\sim 0.1 \mathrm{~nm}$.

It is known that in LIBS measurements the collection geometry is very important and that the plasma expands mostly normal to the sample surface. The on-axis plasma light collection is less sensitive to any changes in the distance between the plasma plume and the collecting F-Theta lens that occur when a groove is processed [33]. In our experiment, the plasma radiation collection angle was chosen to be perpendicular to the sample surface.

Several different scanning algorithms were used for laser microprocessing. Various scanning speeds, pulse repetition rates, thicknesses of the water layer and spacing between the processed grooves were employed for laser cutting and online LIBS signal observation.

\section{Results and discussions}

The experimentation consisted of four main parts: measurement of the laser-induced plasma plume spectrum for different scanning speeds at a different number of passes; measurement of LIBS signals by changing the position of the sample in respect to the focusing F-Theta lens; monitoring the depth of the processed grooves by measuring the LIBS signal and acquiring LIBS signals by changing the thickness of the water layer above the sample surface.

\subsection{LIBS signal at different scanning speeds}

One of the strongest emission lines (Na I at $589 \mathrm{~nm}$ ) in the spectrum of analyzed soda-lime glass was monitored using various scanning algorithms, number of scans, scanning speeds, distance to nominal focal positions and thickness of water layer above the sample surface.

As can be seen in Figure 2, the LIBS signal from material immersed in water at pulse energy $\sim 100 \mu \mathrm{J}$ is a few times weaker than in air, but could be easily resolved and shows the same behavior of the emitted lines and some difference in continuum signal. 


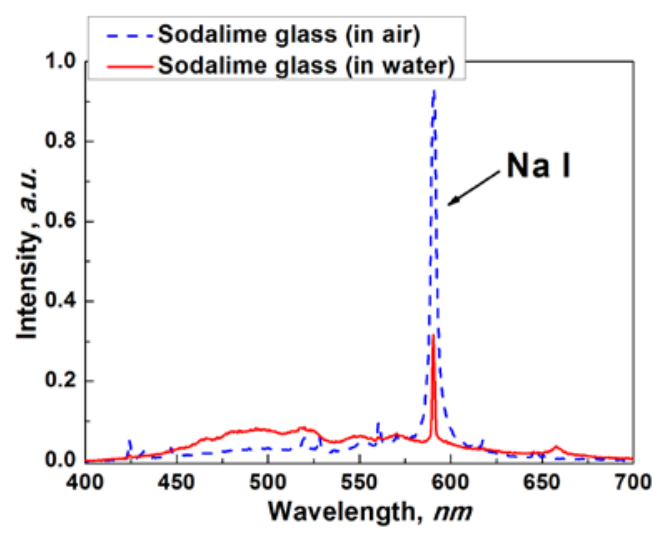

Fig. 2 Emission spectra of soda lime glass sample. Both spectra were obtained using the same energy laser pulses.

Several different scanning algorithms were used for soda-lime processing and LIBS signals were observed. Two different algorithms were used corresponding to $600 \mu \mathrm{m}$ and $200 \mu \mathrm{m}$ spacing between the grooves (Fig. 3). In the case of the scanned picture with line separation of $0.6 \mathrm{~mm}$ for a scanning speed of $100 \mathrm{~mm} / \mathrm{s}$, the time interval between LIBS measurements was equal to $4.2 \mathrm{~s}$, and for a scanning speed of $1000 \mathrm{~mm} / \mathrm{s}$ was equal to $0.412 \mathrm{~s}$. In the case of the scanned picture with line separation of $0.2 \mathrm{~mm}$, the time interval between LIBS measurements for a scanning speed of $100 \mathrm{~mm} / \mathrm{s}$ was equal to $9.8 \mathrm{~s}$, and for a scanning speed of $1000 \mathrm{~mm} / \mathrm{s}$ was equal to $0.98 \mathrm{~s}$. Time intervals between LIBS measurements for the scanned picture with line separation of $0.6 \mathrm{~mm}$ are close to the time intervals between LIBS measurements for the scanned picture with line separation of $0.2 \mathrm{~mm}$ at double the scanning speed. The microgroove geometry was chosen because of the convenience in measuring the depth and width of the groove.

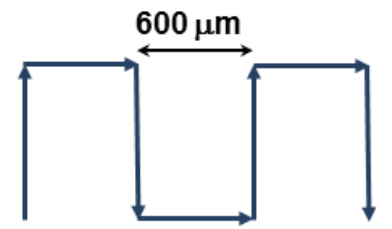

a)

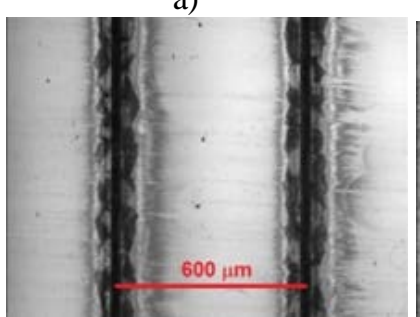

c)

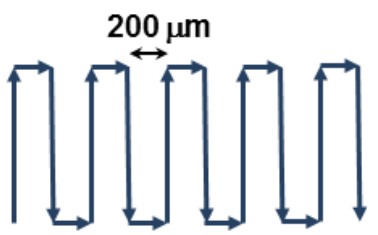

b)

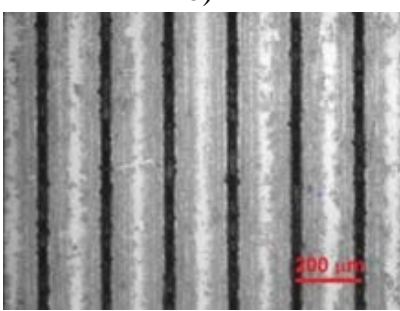

d)
Fig. 3 Schematic view (a, b) and top view (c, d) of the grooves processed using two different scanning algorithms: 600 $\mu \mathrm{m}$ and $200 \mu \mathrm{m}$ gaps between the grooves.

Figure 4(a) shows that LIBS signals decay faster and have no secondary increase of intensity using the scanning algorithm when spacing between the processed grooves was $600 \mu \mathrm{m}$. Figure 4(b) represents spectra that were taken using $200 \mu \mathrm{m}$ spacing between the grooves.
Each data point shown in Fig. 4 is an average value obtained from 4 separate measurements, each made at a new position on the same glass sample by shifting the scanned picture center by $15 \mathrm{~mm}$. The scanned picture looked like a continuous broken line consisting of long parallel and short perpendicular segments. For line separation of $0.6 \mathrm{~mm}$, the scanned picture with dimensions $10 \mathrm{~mm}$ x $11.4 \mathrm{~mm}$ consisted of 20 parallel $10 \mathrm{~mm}$-long lines connected at the bottom or at the top by the next parallel line. For a line separation of $0.2 \mathrm{~mm}$, the scanned picture with dimensions $10 \mathrm{~mm} \mathrm{x}$ $9.6 \mathrm{~mm}$ consisted of 48 lines with the same $10 \mathrm{~mm}$ length connected in the same way. The laser beam was scanned from the beginning to the end of the scanned picture and then in the opposite direction. The LIBS signal measurement was synchronized with the beginning of the line and was measured only when beam was scanned from the beginning to the end of the picture. During opposite scanning, the LIBS signal was not measured. The variation of the LIBS signals measured during an integration time of $100 \mathrm{~ms}$ on the same pass in measurements made at separate glass positions were reproducible, and varied by no more than $5 \%$. The LIBS measurement integration time of $100 \mathrm{~ms}$ corresponded to LIBS signal integration from the scanned pass equal to $10 \mathrm{~mm}$ and $100 \mathrm{~mm}$ for scanning speeds 100 $\mathrm{mm} / \mathrm{s}$ and $1000 \mathrm{~mm} / \mathrm{s}$ respectively. The same measurement procedure was used in all our investigations reported in this article. Laser pulse repetition rate was constant and equal to $100 \mathrm{kHz}$. The beam was scanned multiple times using the same algorithm during entire experiment.

a)

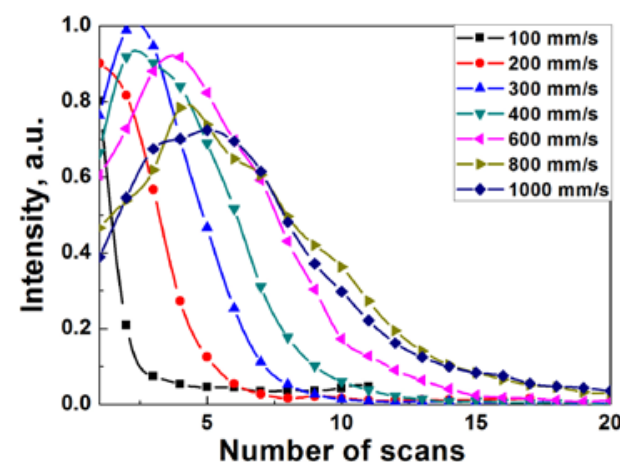

b)

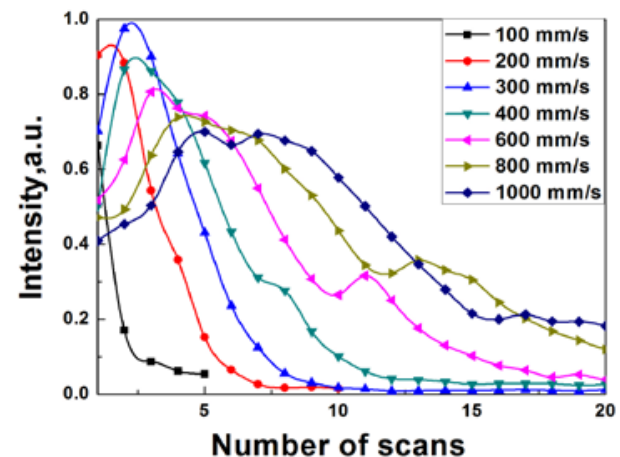

Fig. 4 Na I emission line intensity in relation to the number of scans at seven different scanning speeds. Graphs (a) and (b) show LIBS signal behavior at two different scanning algorithms: $600 \mu \mathrm{m}$ (a) and $200 \mu \mathrm{m}$ (b) spacing between the grooves 
It was determined that narrower spacing between the processed grooves does not provide sufficient irrigation of the processed zone after water layer evaporation in the ablation process, so the intensity increases after several numbers of scans and decays at a slower rate than when using wider spacing between the grooves. LIBS signals decay much faster at a lower scanning speed. This is due to the larger number of laser pulses per scanning length unit, as the laser pulse repetition rate was constant and equal to $100 \mathrm{kHz}$ during this experiment. At a scanning speed of $100 \mathrm{~mm} / \mathrm{s}$, at the spot equal to the focused beam diameter $20 \mu \mathrm{m}$ there is an overlap of 20 pulses, and at a scanning speed of $1000 \mathrm{~mm} / \mathrm{s}$ an overlap of only 2 pulses. When a larger number of pulses with the same energy impinge on the same sample place, a larger amount of the material ablates during a single pass and the ablated groove becomes deeper after a smaller number of scans, and the ablation rate decreases as is usual for deeper holes in drilling experiments [34].

As was mentioned earlier, for a line separation of 0.2 $\mathrm{mm}$ the scanned picture with dimensions $10 \mathrm{~mm}$ x $9.6 \mathrm{~mm}$ consisted of 48 lines, and the time intervals between LIBS measurements for scanning speeds between $600 \mathrm{~mm} / \mathrm{s}$ and $1000 \mathrm{~mm} / \mathrm{s}$ were in the range of $1.6-1 \mathrm{~s}$. This time interval was not sufficient for the thin water layer to fill the whole area with dimensions of $10 \mathrm{~mm} \times 9.6 \mathrm{~mm}$ when it is evaporated by the ablated surface. For line separation of $0.6 \mathrm{~mm}$, the water is evaporated at the line positions but could be refilled from the intermediate zone between the lines. Insufficient irrigation of the scanned zone with a line separation of $0.2 \mathrm{~mm}$ was observed visually. When an additional air fan was used, or the glass sample was fixed incorrectly, water layer disturbance (waving) was observed and this also could contribute to this phenomenon.

\subsection{Focus positioning}

Optimal focusing of the beam is very important for microprocessing the materials and sometimes it has to be monitored and changed in order to get the best results. Therefore, the next experimental step was to determine how the LIBS signal changes at different distances between the focusing F-Theta lens and the sample surface.

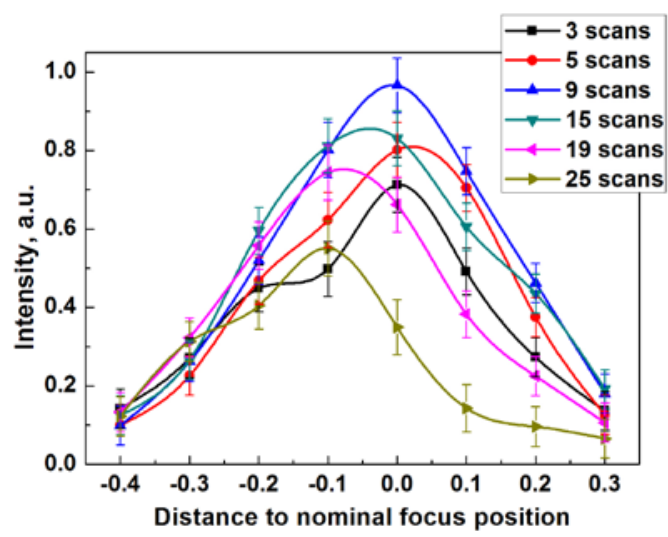

Fig. 5 Intensity of Na I emission line from LIBS spectra of soda-lime glass at different distances to nominal focus positions.

During laser microprocessing, the distance was changed in controlled steps and the LIBS signal was obtained simultaneously. The laser beam scanning speed was $600 \mathrm{~mm} / \mathrm{s}$.
Each data point was taken only during the last scan of algorithm. The Na I emission line in the spectrum of soda-lime glass was monitored for different focusing distances (Fig. 5) As expected, maximum LIBS intensity was reached when the nominal focus position was on the sample surface or slightly, up to $100 \mu \mathrm{m}$, below the sample surface. Analysis of the LIBS signal can therefore be used to estimate the best focusing conditions prior and during laser microprocessing.

\subsection{Monitoring the depth of the processed grooves}

Monitoring the depth of the processed grooves is very important for laser microprocessing. One of the possible applications of LIBS could be monitoring the groove depth of any kind of material. Figure 6 (a) represents how groove depth depends on the number of laser scans on the sodalime glass sample at three different scanning speeds. In order to evaluate the uncertainty in depth measurement, the depth was measured at three different cross-sections along the length of the groove. The pulse repetition rate was constant and equal to $100 \mathrm{kHz}$ for all three scanning regimes. It is obvious that ablation goes faster and grooves become deeper at a lower scanning speed due to the greater number of laser pulses during each scan. At a scanning speed equal to $300 \mathrm{~mm} / \mathrm{s}$ and a repetition rate $100 \mathrm{kHz}, 6.7$ laser pulses overlap, whereas at a scanning speed equal to $600 \mathrm{~mm} / \mathrm{s}$, only 3.3 pulses overlap.

a)

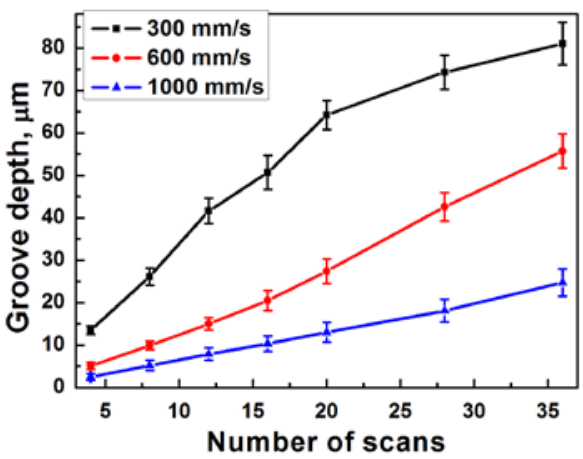

b)

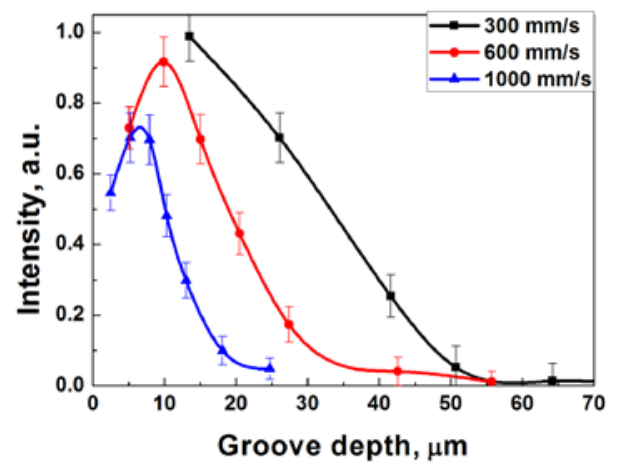

Fig. 6 Groove depth after a certain numbers of scans (a) and Na I spectral line intensity variation in relation to groove depth (b)

At the beginning, the groove depth linearly depends on the number of scans, and after it reaches $60 \mu \mathrm{m}$ saturation occurs. Using a higher scanning speed, the depth of the grooves increases considerably more slowly, and therefore saturation is not observed after the same number of laser scans. Figure 6 (b) represents the intensity of $\mathrm{Na}$ I spectral 
line variation in relation to the groove depth. Depending on the scanning speed, the maximum intensity is observed when the groove depth is in the range of 8 to $13 \mu \mathrm{m}$, and it further rapidly decreases after the grooves become deeper. This could probably be related to the increase in laser radiation absorption when a crater with a depth-to-width ratio from 0.4 to 0.65 is formed. A further increase in depth must also affect larger light absorption, but this could be prevented by decreasing the number of radiation emitting species, which could leave deeper grooves.

The saturation in Fig. 6 b occurred due to the selected measuring procedure, as the LIBS signal in this case was measured on the 4th, 8th, 12th, 16th, and 20th scans to cover the large range of groove depths. There were even larger LIBS signal values at earlier scans, but this was not detected in this case by the measurement algorithm used. The LIBS signal increases with laser pulse energy and laser power when the energy fluency is sufficient for ablation. For large LIBS signals, saturation can be avoided by using a smaller integration time or by additional attenuation of the LIBS radiation.

The scanning speed had a minimal impact on the groove width. Similarly to the depth, the groove width was also measured by three different cross-sections along the groove length. The variation in widths measured for different scanning speeds (in the range from $300 \mathrm{~mm} / \mathrm{s}$ to $1000 \mathrm{~mm} / \mathrm{s}$ ) at the same number of scans was within $15 \%$. Therefore, the groove width in this scanning speed range is mainly influenced by the geometric spot size of the focused laser beam. A different situation was observed at scanning speeds lower than $200 \mathrm{~mm} / \mathrm{s}$, when the groove widths increased. This could be explained by the fact that, after a series of overlapping pulses, water is evaporated from the surface of the sample faster than it can be replenished. If this occurs, material is being removed via ablation without water, thus giving wide groove profiles.

\subsection{Influence of the thickness of the water layer}

As described above, the water layer on the surface of the sample acts as a buffer layer where filamentation appears. Due to this water layer, thermal effects are reduced and the debris from the processed surface is removed. The thickness of the water layer on the surface of the glass has a significant influence on groove quality and depth.

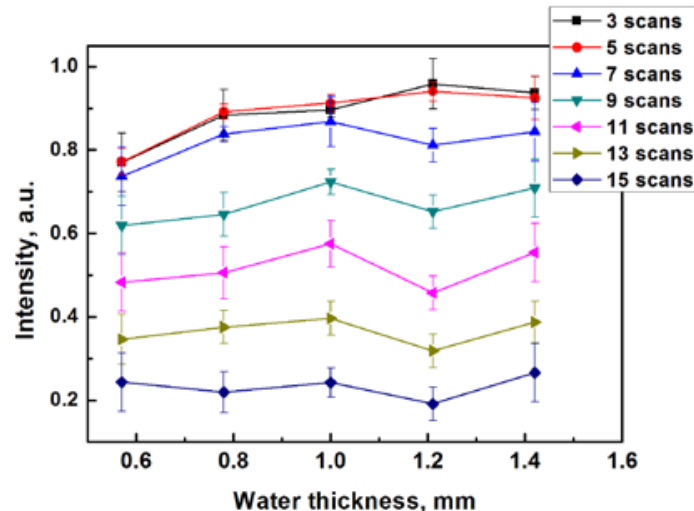

Fig. $7 \mathrm{Na}$ I emission line intensity after n-th scans in relation to water layer thickness.
Figure 7 shows how Na I emission line intensity depends on the thickness of the water layer on the sample surface. Three measurements were taken for each data point. In this case LIBS signal corresponds to the radiation emitted during some part of the n-th scan. The groove depth was estimated after 13 laser passes over the sample surface. As in the section above, depth was measured at three different cross-sections along the length of the groove.

In order to compare the groove depth after 13 scans with the corresponding LIBS signal, the integrated LIBS signal after 13 scans in dependence on the thickness of the water layer on the sample surface was evaluated (Figure 8).

a)

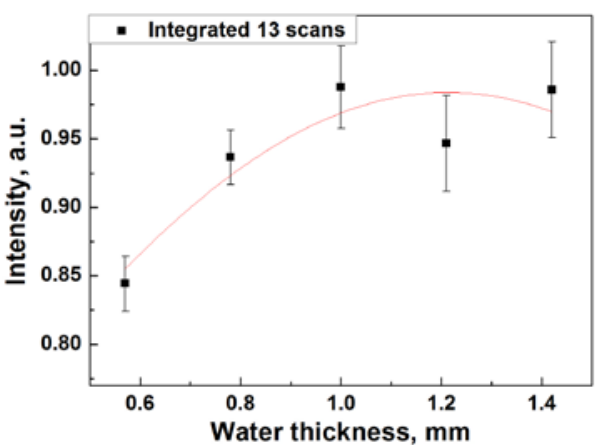

b)

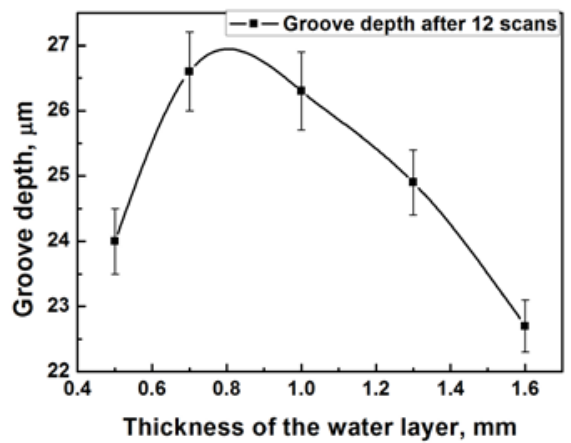

Fig. 8 Integrated LIBS signal (Na I emission line) intensity (a) and groove depth after 13 scans (b) in relation to water layer thickness (scanning speed was constant and equal to $400 \mathrm{~mm} / \mathrm{s}$ ).

The curve in Fig. 8 b was obtained by investigating the depth of the ablated grooves with an optical profilometer, and this showed the largest depths for water thicknesses in the range between $0.7-1 \mathrm{~mm}$. This water layer corresponds to the best conditions for fastest ablation. Some correlation was found between the depth of the ablated grooves and the LIBS signal in relation to the water layer thickness. The LIBS signal drastically decreased for water thicknesses greater than $2 \mathrm{~mm}$ (not included in Fig. 8 a) and this was related with a non-evaporated layer of water during the ablation process, which introduced large nonlinear losses for laser pulses reaching the glass sample, bubble formation in the remaining water layer, and small production of excited species in the ablation plume. The largest integrated LIBS signals and the largest amount of excited species produced by the LIBS signal were obtained for water thicknesses in the range of $0.9-1.3 \mathrm{~mm}$. Integration of the LIBS signals in every case, but not every second case as was done in our 
experiments, would probably give an even better correlation with groove depth.

It can be seen from the ablated grooves depth measurements that the optimal thickness of the water layer and the most effective ablation occurs when the thickness of the water layer is in the range of 0.7- $1 \mathrm{~mm}$. Similar behaviour in the integrated LIBS signal dependence on the water layer thickness was observed in the LIBS experiments.

\section{Conclusion}

Laser micromachining related with ablation can benefit from laser-induced breakdown spectroscopy, as not only the chemical composition can be determined, but an insight can also be gained into the instant characteristics or quality of the micromachining. In this report we presented results on the application of LIBS as a monitoring tool for the micromachining of materials immersed in water and processed with femtosecond high repetition rate pulses. LIBS signals decay faster and have no secondary increase of intensity, which is related with water disappearance and ablation in air using a scanning algorithm with wider spacing between the grooves. Narrower spacing between the processed grooves does not provide sufficient irrigation of the processed zone to achieve fully immersed target ablation conditions, so the intensity increases after several scans and decays at a slower rate than when a wider spacing between the grooves is used. This effect is more conspicuous at higher scanning speeds. The most intensive signal is obtained using the distance equal to nominal focal length between the sample and focusing optics. Therefore it can be used as a tool for adjusting the distance to sample for laser micromachining. Spectral line emission and depth of the groove dependence shows that maximum intensity is reached at about 8-10 $\mu \mathrm{m}$ below the sample surface and further proportionally decreases as the depth of the groove increases. Moreover the most effective ablation occurs when the thickness of the water layer is in the range of $0.7-0.1 \mathrm{~mm}$. The integrated LIBS signal intensity correlated with the measured groove depths after 13 scans.

This particular study demonstrates that, during the optimization of the laser micromachining process, some correlations in the LIBS spectra can be observed and used for the implementation of laser processing monitoring.

\section{Acknowledgments}

This project has received funding from the European Union's Horizon 2020 research and innovation programme under grant agreement no 654148 Laserlab-Europe.

\section{References}

[1] K. C. Phillips, H. H. Gandhi, E. Mazur, and S. K. Sundaram: Adv. Opt. Photon., 7, (2015) 684.

[2] S. K. Sundaram, E. Mazur: Nat. Mater., 1, (2002) 217.

[3] Y. Shimotsuma, K. Hirao, P.G. Kazansky, J. Qiu: Jpn. J. Appl. Phys., 44, (2005) 4735.

[4] R.R. Gattass, E. Mazur: Nat. Photon., 2, (2008) 219.

[5] K. Sugioka, Y. Cheng: Light: Sci. Appl., 3, (2014) e149.

[6] W. Wiesemann, Process monitoring and closed-loop control, in: Landolt-Bornstein, Laser Physics and Applications, Vol. 1C, (2004) S. 243-275.
[7] J.M. Lee, K.G. Watkins: Optics and Lasers in Engineering, 34, (2000) 429.

[8] W. M. Steen: "Laser material processing”, (Springer, London, 2003) p. 533.

[9] M. K. Jeon, W. B. Kim, G.C. Han, S.J. Na: Journal of Materials Processing Technology, 82, (1998), 53.

[10] J. Beersiek, T. Devermann, K. Behler: Proc. 23rd Int. Congress on Applications of Lasers and ElectroOptics (2004) p. 1001.

[11] A. Stournaras, K. Salonitis, G. Chryssolouris: The International Journal of Advanced Manufacturing Technology, 46, 5-8 (2010) 589.

[12] Pandey ND, Shan HS, Barti A: Int. J. Adv. Manuf. Technol., 28, (2006) 863

[13] A. Sun, E. Kannatey, Jr., M. Gartner: Journal of Laser Applications 14, 2 (2002) 114.

[14] I. Gobernado-Mitre, A.C. Prieto, V. Zafiropulos, Y. Spetsidou and C. Fotakis: Appl. Spectrosc., 51, (1997) 1125.

[15] T.J. Li, Q.H. Lou, Y.R. Wei, F.Huang, J.X. Dong, and J.R. Liu: Appl. Surf.Sci., 181, (2001) 225.

[16] T. Tong, J. Li, J.P. Longtin: Appl. Opt., 43, 9, (2004) 1971.

[17] J.O. Connolly, G.J. Beirne, G.M. O’Connor, T.J. Glyn, A.J. Conneely: Proc. of SPIE 3935 (2000), p. 132.

[18] T. Sibillano, A.Ancona, V. Berardi, P.M. Lugara: Sensors, 9, (2009) 3376.

[19] D. Diego-Vallejo, M. Schwagmeier, D. Ashkenasi, G. Illing, H.J. Eichler: Journal of Laser Micro/Nanoengineering, 6, 2, (2011) 146.

[20] D. Diego-Vallejo, D. Ashkenasi, A. Lemke, H.J. Eichler: Spectrochimica Acta Part B: Atomic Spectroscopy, 87, (2013) 92.

[21] D. Diego-Vallejo, D. Ashkenasi, H.J. Eichler: Proc. Lasers in manufacturing WLT, Munich, Conf. Proc. on Physics Proceedia, 41, (2013) 904.

[22] Cramers, D. "Handbook of Laser-Induced Breakdown Spectroscopy”. John Wiley. 2006.

[23] J.M. Vadillo and J.J. Laserna: Spectrochim. Acta B 59, (2004) 147-161.

[24] T.A. Labutin, A.M. Popov, V.N. Lednev and N.B. Zorov: Spectrochim. Acta B 64, (2009) 938-949.

[25] E. Tzamali and D. Anglos: "Lasers in the Conservation of Artworks" ed. by J. Nimmrichter, W. Kautek and M. Schreiner, (Springer, Heidelberg, 2007) p. 377.

[26] T.V. Kononenko, D. Walter, V.I. Konov and F. Dausinger: Quantum Electron 39, (2009) 328.

[27] H. Balzer, M. Hoehne, R. Noll and V. Sturm: Anal. Bioanal. Chem. 385 (2006), 225.

[28] T.A. Labutin,V. N. Lednev, A. A. Ilyin and A. M. Popov: J. Anal. At. Spectrom. 31, (2016), 90.

[29] S. Butkus, D. Paipulas, R. Sirutkaitis, E. Gaižauskas and V. Sirutkaitis: Journal of Laser Micro/Nanoengineering 9(3), (2014) 213.

[30] S. Butkus, E. Gaižauskas, D. Paipulas, Ž. Viburys, D. Kaškelytė, M. Barkauskas, A. Alesenkov, V. Sirutkaitis: Appl. Phys. A, 114(1), (2014) 81.

[31] S. Butkus, A. Alesenkov, E. Gaižauskas, D. Paipulas, D. Kaškelytė, M. Barkauskas, V. Sirutkaitis, Micromachines, 6, 2010-2022 (2015). 
[32] E. Gaižauskas, A. Dubietis, V. Kudriašov, V. Sirutkaitis, A. Couairon, D. Faccio, P. Di Trapani, in: R. W. Boyd et al. (eds), Self-focusing: Past and Present 457-479, (Springer, 2009).

[33] E. Tognoni, V. Palleschi, M. Corsi, G. Cristoforetti: Spectrochimica Acta Part B 57, (2002) 1115.

[34] A. Ruf, D. Breitling, C. Fohl, J. Radtke, F. Dausinger, T. Konenenko, S. Klimentov, S. Garnov, V. Konov, J. Suzuki, in Wissenschaftliche Gesellschaft Lasertechnik $\{$ WLT $\}$ e.V.(Ed.):Proc. First Int. WLTConference on Lasers in Manufacturing (ATFachverlag, Stuttgart 2001) pp. 214-226.

(Received: June 7, 2016, Accepted: October 6, 2016) 